\title{
Gas dynamics in whole galaxies: $\mathrm{SPH}$
}

\author{
Clare Dobbs ${ }^{1,2}$ \\ ${ }^{1}$ Max-Planck-Institut für extraterrestrische Physik, \\ Giessenbachstraße, D-85748 Garching, Germany \\ email: cdobbs@mpe.mpg.de \\ ${ }^{2}$ Universitäts-Sternwarte München, Scheinerstraße 1, D-81679 München, Germany
}

\begin{abstract}
I review the progress of SPH calculations for modelling galaxies, and resolving gas dynamics on GMC scales. SPH calculations first investigated the response of isothermal gas to a spiral potential, in the absence of self gravity and magnetic fields. Surprisingly though, even these simple calculations displayed substructure along the spiral arms. Numerical tests indicate that this substructure is still present at high resolution (100 million particles, $\sim 10 \mathrm{pc})$, and is independent of the initial particle distribution. One interpretation of the formation of substructure is that smaller clouds can agglomerate into more massive GMCs via dissipative collisions. More recent calculations have investigated how other processes, such as the thermodynamics of the ISM, and self gravity affect this simple picture. Further research has focused on developing models with a more realistic spiral structure, either by including stars, or incorporating a tidal interaction.
\end{abstract}

Keywords. ISM: clouds, galaxies: ISM, galaxies: spiral, stars: formation, hydrodynamics

\section{Introduction}

The last decade has seen massive progress in the use of numerical calculations to model the ISM, both on galactic and subgalactic scales. Early particle simulations (Levinson \& Roberts 1981; Kwan \& Valdes 1983, 1987; Hausman \& Roberts 1984; Tomisaka 1984, 1985; Roberts \& Stewart 1987) were largely limited to modelling individual clouds as ballistic particles. Now, hydrodynamic simulations of galaxies are able to resolve GMCs. Furthermore they can capture the dynamical evolution of molecular (and atomic) clouds, as they form, interact and disperse.

To maximise resolution, hydrodynamic simulations frequently apply an external potential, which includes the dark matter halo and stellar disc. Numerous calculations have also included a spiral component to the stellar potential in order to simulate a grand design galaxy. Surprisingly, calculations which model the gas response to a spiral potential show the presence of substructure in the gas, even without magnetic fields or self gravity (Wada \& Koda 2004, Dobbs \& Bonnell 2006). This substructure becomes more clearly visible as the spiral arm clumps are sheared out into spurs, or feathers, in the interarm regions. Such structure is found to occur in both grid (Wada \& Koda 2004, Kim \& Ostriker 2006) and SPH (Wada \& Koda 2004, Dobbs \& Bonnell 2006) codes.

Wada \& Koda 2004 interpreted the formation of substructure along the spiral arms in terms of Kelvin Helmholtz instabilities. However Dobbs et al. 2006 provided a different explanation for the formation of spiral arm clumps. They likened the passage of gas through the spiral shock to a queue of traffic, where gas particles (or clumps) become bunched together. This occurs because the gas particles undergo dissipative collisions in the spiral arms. This process resembles prior models of cloud coalescence, but the accretion and dispersal of gas into and from clumps is more continuous. Also, there is no need for the gas to be molecular, although for some environments e.g. M51 it may 
well be. The main requirement (Dobbs \& Bonnell 2006) is that the gas is cold, so that the spiral shock is strong, and the gas pressure (which would cause clouds to diffuse) is minimal. The spacing of the clumps is $\sim \sigma_{v} T$ where $\sigma_{v}$ is the turbulent velocity dispersion of the gas, and $T$ the time spent in the spiral arms (Dobbs, Bonnell \& Pringle 2006, Dobbs 2008). In simulations with higher spiral potentials (and consequently higher arm to interarm contrasts), $T$ increases and the spacing between the clumps increases (Dobbs 2008).

\section{Resolution studies}

A potential issue with the results of these calculations is that the structure may be resolution dependent, or even disappear at high resolution. In Fig. 1, we show this is not the case. Similar structure is apparent with 1, 20 and 100 million particles, the latter corresponding to a mass resolution of $10 \mathrm{M}_{\odot}$ and a spatial resolution of $<1 \mathrm{pc}$. It is difficult to quantify the spacing between the features along the spiral arms - partly because there is no exact periodicity. Nevertheless in Fig. 2, we show the auto-correlation function, taken from the distribution of particles in $\log (r)$ versus $\theta$ space. The position of the first peak indicates the spacing which provides the best correlation. We see that
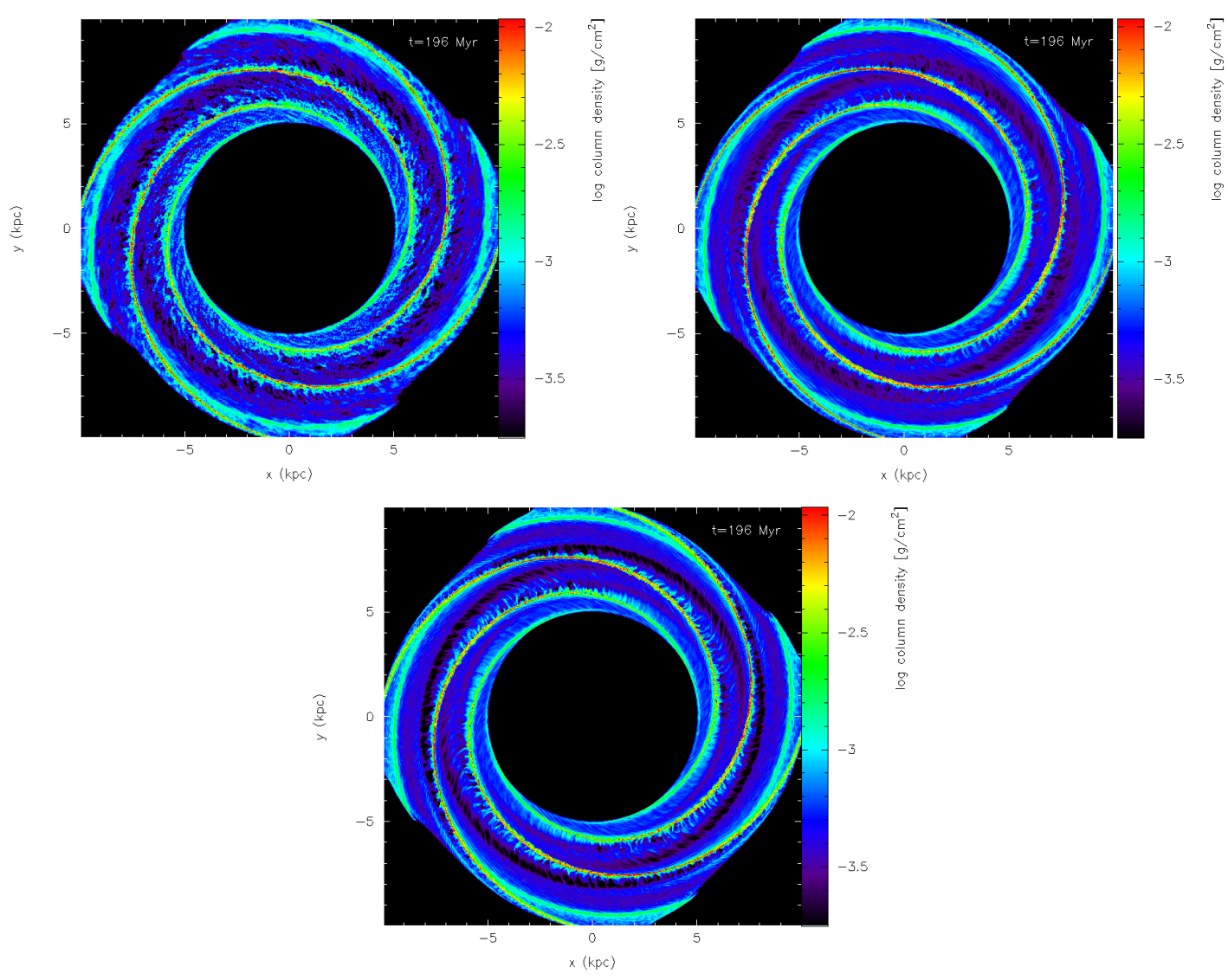

Figure 1. Resolution test for hydrodynamic calculations with a spiral potential alone. The number of particles in each panel is 1 million (top left), 20 million (top right) and 100 million (lower). 


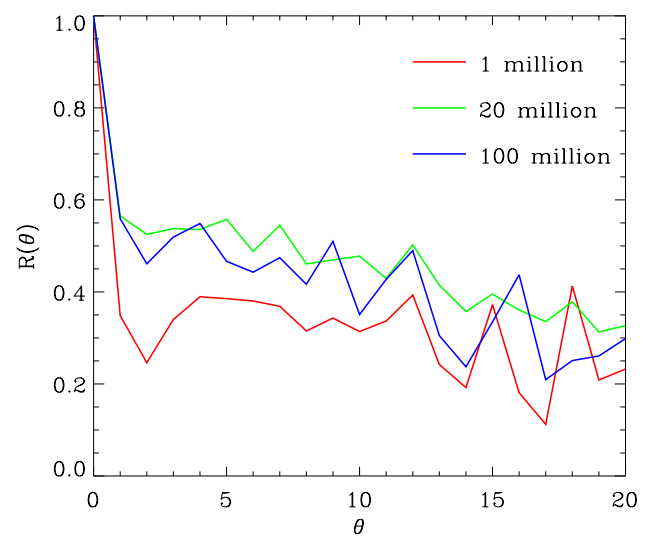

Figure 2. The auto-correlation function $(R)$ is plotted for the distribution of particles along a spiral arm. $\theta$ is the angle, or distance along the spiral arm. The correlation function is the sum of the distribution of particles, multiplied by the distribution shifted by $\theta$. Thus the first peak represents the best-fit separation of the spurs. The similarity in location of the first peaks, and spacing of subsequent peaks implies that the spacing is not resolution dependent. The spacing is around $200 \mathrm{pc}$, but has not yet reached a maximum (which is closer to $700 \mathrm{pc}$ (Dobbs, Bonnell \& Pringle 2006)).

the peak is in a similar location for each resolution, and also that the typical spacing between subsequent peaks is similar. Unfortunately the spacing of the clumps has not reached a maximum at this time (200 Myr) as it was too time consuming to run the 100 million particle simulation further.

In Fig. 3, we show further tests, varying the viscosity and the initial particle distribution. We use 1 million particles in each calculation. The interarm spurs occur in all the cases, and in particular using an initially uniform particle distribution (rather than a random distribution, which is used for all the other calculations) makes negligible difference to the results.

\section{Thermodynamics and molecular hydrogen formation}

Early calculations adopted an isothermal medium (Slyz et al. 2003, Wada \& Koda 2004, Dobbs \& Bonnell 2006), but more recent calculations have included a much more sophisticated treatment of the ISM (Dobbs et al. 2008, Peluppessy \& Papadopoulos 2009). In Dobbs et al. 2008, we used the thermodynamics and chemistry of Glover \& MacLow 2007. The results of these calculations indicate that GMC formation occurs in the same way as isothermal simulations of cold gas, by the coalescence of clouds in the spiral shock, as most of the gas (70\%) tends to be cold.

By including $\mathrm{H}_{2}$ formation, we can start to make estimates on cloud formation timescales, and cloud lifetimes. In Dobbs et al. 2008, we found $\mathrm{H}_{2}$ forms on timescales of Myrs, whilst once formed, $\mathrm{H}_{2}$ lasts 10's of Myrs. However these calculations do not include any feedback processes, only $\mathrm{H}_{2}$ photodissociation. Lifetimes based around the cloud dynamics, rather than $\mathrm{H}_{2}$ fractions, are difficult to determine, since the clouds evolve dynamically on relatively short timescales.

The inclusion of $\mathrm{H} 2$, and $\mathrm{CO}$ also allows the opportunity of comparison with observations. Pelupessy \& Papadopoulos 2009 (Fig. 5) calculate synthetic UV and CO maps of 

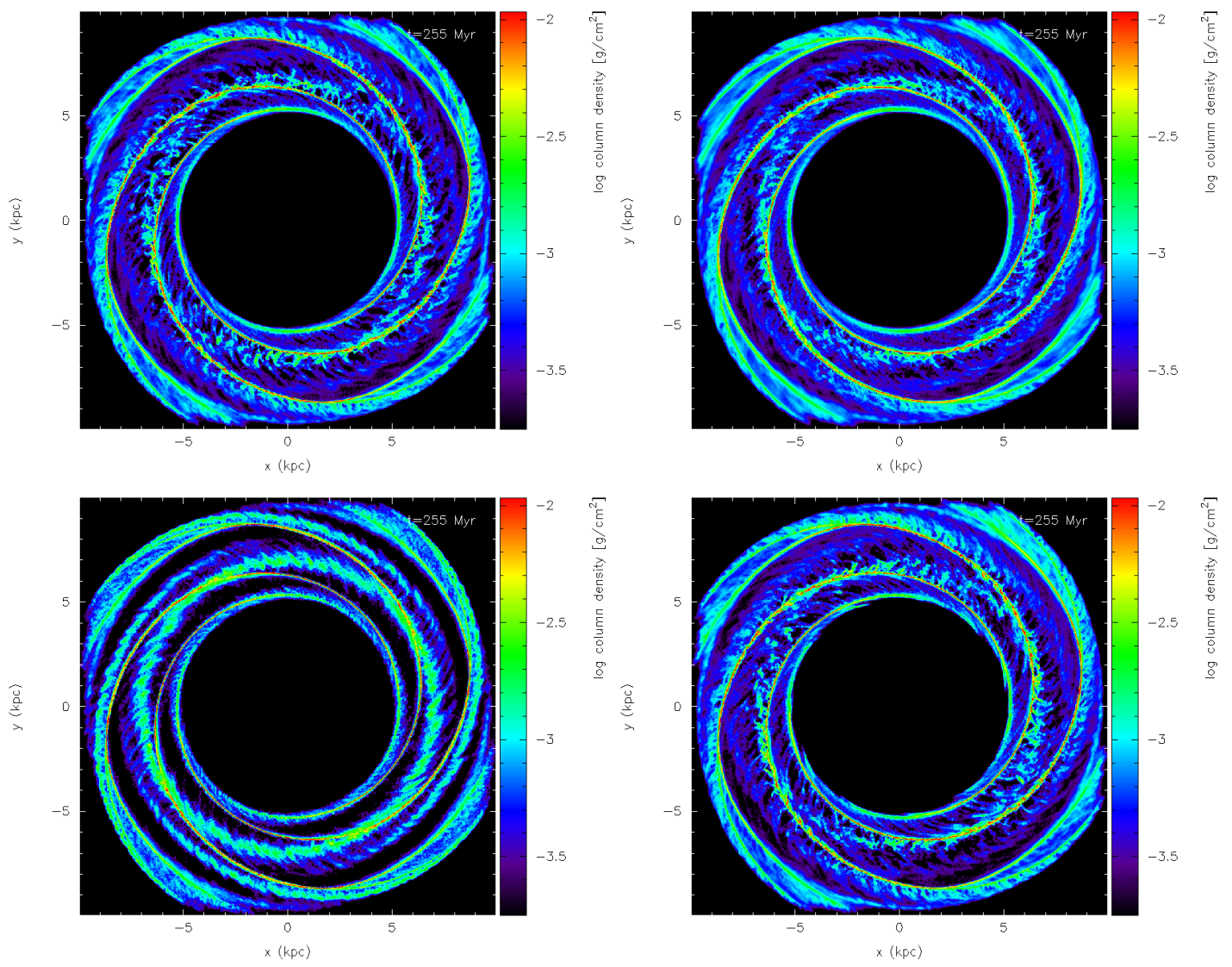

Figure 3. Column density images from SPH simulations of isothermal gas subject to a spiral potential. Three plots show different artificial viscosity settings: the standard case, $\alpha=1, \beta=2$ (top left); $\alpha=2, \beta=4$ (top right); Balsara switch with $\alpha=1, \beta=2$ (lower left). Finally the lower right panel shows the column density when the particles are initially set up uniformly, on concentric circles. The difference for the Balsara switch is probably because the shock is not captured so well.

their galaxy models. In Douglas et al. 2010, we present synthetic HI maps, which show abundant HI self absorbtion features (also Acreman et al., these proceedings).

\section{Magnetic fields}

Magnetic fields have recently been implemented in simulations of galaxies (Dobbs \& Price 2008, Dolag \& Stasyszyn 2008, Kotarba et al. 2009) using the Euler potential method (Price, these proceedings). Although the current Euler potential implementation is known to have limitations, in particular amplification of the field due to winding is not captured, this method does allow a first order study of the effects of magnetic fields.

In Dobbs \& Price 2008 we find that the main effect of the magnetic fields is to contribute pressure to the ISM. The result is similar to increasing the thermal pressure, as the spiral arms and spurs are less dense and more diffuse. However spurs are still visible for plasma $\beta>0.1$. Kotarba et al. 2009 study the amplification of magnetic fields in SPH simulations with stars and gas, where the spiral arms arise consistently. They compare 

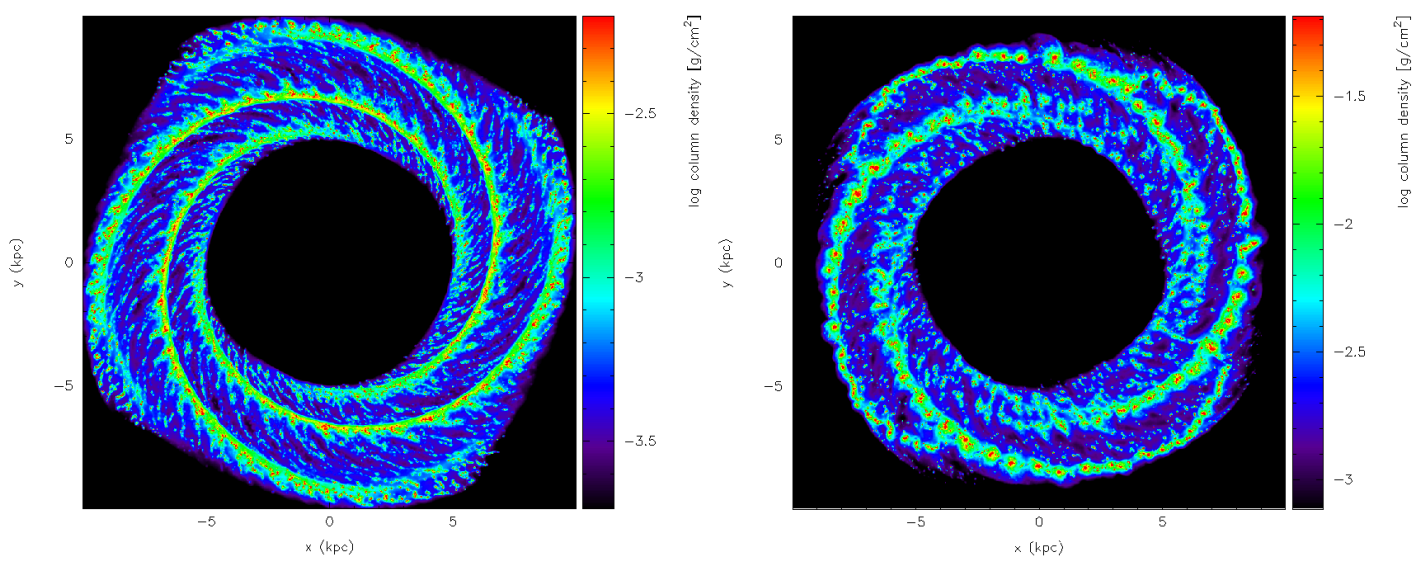

Figure 4. Column density from simulations with surface densities of $4 \mathrm{M}_{\odot} \mathrm{pc}^{-2}$ (left) and 20 $\mathrm{M}_{\odot} \mathrm{pc}^{-2}$ (right). The simulations include self gravity and magnetic fields, adopting an isothermal two-phase medium of cold and warm gas. Self gravity has little effect on the global structure in the low surface density case, but aids the formation of more massive complexes in the higher density case.

calculations with the Euler method, and direct calculation of B, finding that even though the amplification is likely underestimated with the Euler method, the large value of div $B$ when using the direct calculation of $B$ render this method much less reliable.

\section{Self gravity}

In Dobbs 2008, we perform simulations with self gravity and magnetic fields. Self gravity enhances the formation of molecular clouds, by increasing the frequency and success (i.e. the likelihood that clouds merge rather than get disrupted) of collisions. Self gravity also leads to gravitational instabilities in the gas. The net result is that clouds reach higher densities compared to the ambient medium, and are easier to distinguish along the spiral arms. The difference in structure becomes more noticeable with increasing surface densities - the gas distribution when $\Sigma=4 \mathrm{Mpc}^{-2}$ is similar whether or not self gravity is included, but self gravity has a much stronger impact on the global structure when $\Sigma=20 \mathrm{Mpc}^{-2}$ (Fig. 4). The surface density in the solar neighbourhood lies in between these regimes (Wolfire et al. 2003).

\section{Feedback}

So far the effect of feedback on molecular cloud formation, and the evolution of spurs has not been studied for grand design galaxies with SPH. This has been studied with grid codes, e.g. Wada 2008 find that supernovae have a secondary effect, and spurs are still evident.

\section{SPH simulations without a spiral potential}

Several authors have also modelled flocculent galaxies, where the structure is solely due to gravitational and thermal instabilities, and is presented elsewhere in these proceedings (Wada).

Pelupessy \& Papadopoulos (2006,2009) also model flocculent spirals (Fig. 5), and show 
that they are able to reproduce characteristics of the ISM such as PDFs and the pressure $\mathrm{H}_{2}$ relation (Blitz \& Rosolowsky 2004) reasonably well. They also use the amount of $\mathrm{H}_{2}$ formation to estimate the star formation efficiency, which is then used in their models of feedback. One of their main results is that for gas rich, and metal poor galaxies, they find strong deviations from the star formation rates in their models and those predicted from the Schimdt-Kennicutt relation (Pelupessy \& Papadopoulos 2010).

Alternatively a grand design spiral pattern can be obtained more realistically by modelling an interaction with another galaxy (see Struck, these proceedings). In Dobbs et al. 2010, we modelled the grand design spiral M51 (Fig. 6), and showed that the interaction with the companion can reproduce both the observed spiral pattern, and detailed features in the gas. We also found the pattern highly time dependent. Other studies have concentrated on reproducing the star formation histories of interacting galaxies, e.g. Karl et al. 2010.

\section{Properties of molecular clouds formed in the simulations}

In Dobbs 2008, we started to determine properties of molecular clouds formed in the simulations with a fixed spiral potential. The mass functions of the clouds have an index of 1.7-2.0, in line with observations. The cloud angular momenta also appear in good agreement with clouds in both the Milky Way (Phillips 1999) and M33 (Rosolowsky et al. 2003). Clumps regularly undergo collisions or interactions with other clumps, which can lead to clumps rotating in a retrograde direction, i.e. in the opposite sense to galactic rotation. Properties of molecular clouds have also been deteremined in calculations which
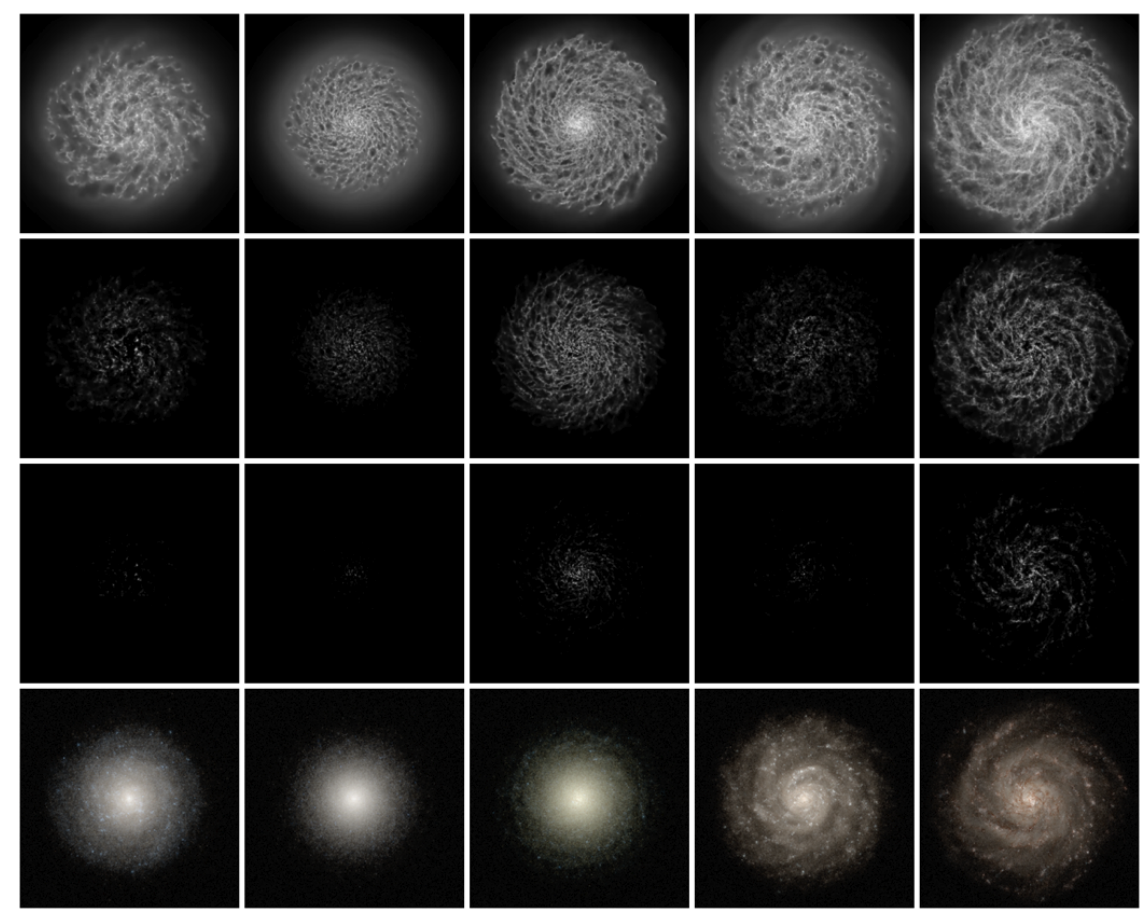

Figure 5. Column density images from Pelupessy \& Papadopoulos 2009, for simulations of flocculent spirals, which include the gas and stars. The images show HI (top), $\mathrm{H}_{2}$ (second line), CO (third line) and UBV (lowest line) from models with varying amounts of gas and metallicites. 


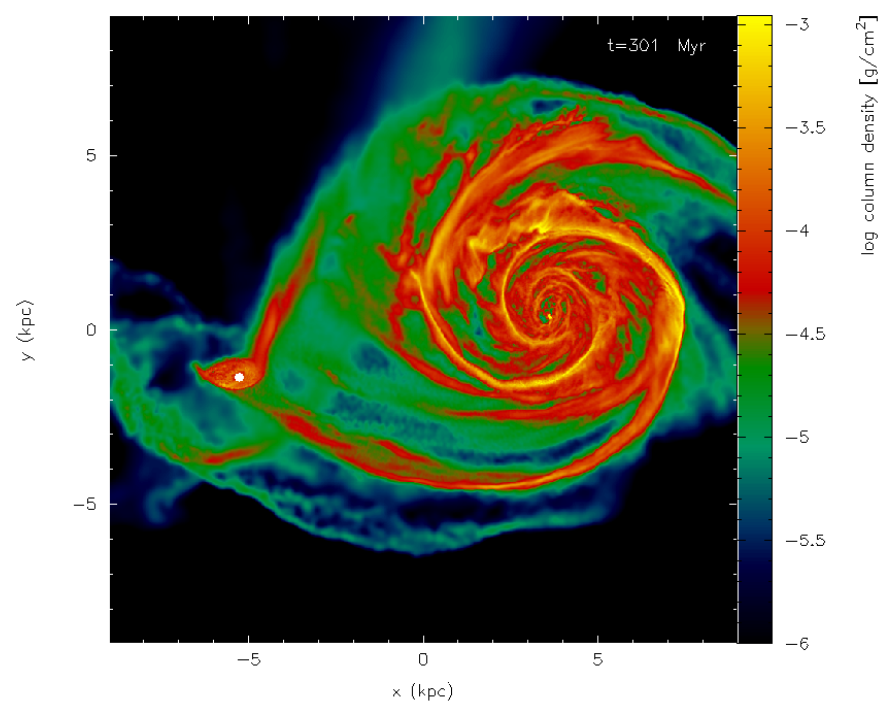

Figure 6. This figure is taken from a calculation designed to reproduce the spiral structure of M51 (Dobbs et al. 2010). The companion galaxy, NGC 5193, is modelled as a point mass (seen as a white dot in the figure), and the two galaxies are given inital velocities and positions derived using N-body calculations (Theis \& Spinneker 2003).

used the AMR code ENZO (Tasker \& Tan 2009), and show remarkable similarity with $\mathrm{SPH}$ calculations, although there are a number of differences between the models (e.g. presence of magnetic field, spiral potential).

Alves (these proceedings) derive a remarkably tight mass radius relation for molecular clouds. This very tight correlation is not apparent in the results of Heyer et al. 2009, who re-examined the properties of clouds observed by Solomon et al. 1987. Numerical simulations may help explain the discrepancies in these results, and why the clouds found by Heyer et al. 2009 tend to be slightly unbound.

\section{Conclusion}

SPH calculations allow the ISM and molecular clouds to be studied on galactic scales. Unlike previous particle methods, these calculations can model individual clouds, and thus capture changes in the size, shape, and constituent gas of the clouds. Hydrodynamic simulations show that when a global grand design spiral pattern is present, GMCs form by the agglomeration of gas clouds in the spiral shock. This process requires the gas is clumpy (or cold), but appears independent of resolution down to at least $10 \mathrm{M}_{\odot}$ per particle. It is also independent of the treatment of artificial viscosity, and the initial particle distribution. Magnetic fields do not strongly affect this picture, and the detailed thermodynamics do not make a significant difference, provided there is cold gas. Self gravity can alter the large scale distribution, both enhancing cloud agglomeration and gravitational instabilities, the importance of self gravity increasing with surface density.

\section{Acknowledgments}

I would like to thank Ian Bonnell and Jim Pringle for their help throughout this research. I also thank Daniel Price for providing his SPH code 'phantom' which was used 
to perform the resolution test in Section 2. The calculations reported here were performed using the University of Exeter's SGI Altix ICE 8200 supercomputer, and the HLRB-II: SGI Altix 4700 supercomputer at the Leibniz supercomputer centre, Garching. Images included in this review were produced using SPLASH (Price 2007), a visualisation tool for SPH that is publicly available at http://www.astro.ex.ac.uk/people/dprice/splash.

\section{References}

Blitz, L. \& Rosolowsky, E. 2004, ApJL, 612, 29

Dobbs, C. L. \& Bonnell, I. A. 2006, MNRAS, 367, 873

Dobbs, C. L., Bonnell, I. A. \& Pringle, J. E. 2006, MNRAS, 371, 1663

Dobbs, C. L. 2008, MNRAS, 391, 844

Dobbs, C. L. \& Price, D. J. 2008, MNRAS, 383, 497

Dobbs, C. L., Glover, S. G., Clark, P. C. \& Klessen, R. S. 2008, MNRAS, 389, 1097

Dobbs, C. L., Theis, C., Pringle, J. E. \& Bate, M. R. 2010, MNRAS, 403, 625

Dolag, K., \& Stasyszyn, F. 2009, MNRAS, 398, 1678

Douglas, K., Acreman, D., Dobbs, C. \& Brunt, C. 2010, MNRAS, tmp, 896

Glover, S. G. \& Mac Low, M. M. 2007, ApJ, 169, 239

Hausman, M. A. \& Roberts, W. W. Jr 1984, ApJ, 282, 106

Heyer, M., Krawczyk, C., Duval, J. \& Jackson, J. M. 2009, ApJ, 699, 1092

Karl, S. J., Naab, T., Johansson, P. H., Kotarba, H., Boily, C. M., Renaud, F. \& Theis, C. 2010, ApJ, 715, 88

Kim, W.-T. \& Ostriker, E. C. 2006, ApJ, 646, 213

Kotarba, H., Lesch, H., Dolag, K., Naab, T., Johansson, P. H., \& Stasyszyn, F. A. 1983, MNRAS, 397,733

Kwan, J. \& Valdes, F. 1983, ApJ, 271, 604

Kwan, J. \& Valdes, F. 1987, ApJ, 315, 92

Levinson, F. H. \& Roberts, W. W. Jr 1981, ApJ, 245, 465

Papadopoulos, P. P. \& Pelupessy, F. I.. 2010, ApJ, 717, 1037

Pelupessy, F. I., Papadopoulos, P. P. \& van der Werf, P. 2006, ApJ, 645, 1024

Pelupessy, F. I. \& Papadopoulos, P. P. 2009, ApJ, 707, 954

Phillips, J. P. 1999, A\&AS, 134, 241

Price, D. J. 2007, PASA, 24, 159

Roberts, W. W. Jr \& Stewart, G. R. 1987, ApJ, 282, 106

Rosolowsky, E., Engargiola, G., Plambeck, R. \& Blitz, L. 2003, ApJ, 599, 258

Slyz, A., Kranz, T. \& Rix, H.-W. 2003, MNRAS, 346, 1162

Solomon, P. M., Rivolo, A. R., Barrett, J. \& Yahil, A. 1987, ApJ, 319, 730

Tasker, E. J., \& Tan, J. C. 2009, ApJ, 700, 358

Theis, C. \& Spinneker, C. 2003, PASJ, 284, 495

Tomisaka, K. 1984, PASJ, 36, 457

Tomisaka, K. 1986, PASJ, 38, 95

Wada, K. \& Koda, J. 2004, PASJ, 38, 95

Wada, K. 2008, ApJ, 675, 188

Wolfire, M. G., McKee, C. F., Hollenbach, D. \& Tielens, A. G. G. M. 2003, ApJ, 587, 278 